\section{Zusammenhang zwischen Arthritis, Inflammation und gutem Schlaf untersucht}

Lee S et al. Arthritis, Sleep Health, and Systemic Inflammation in Older Men. Arthritis Care Res 2020; 72: 965-973
Mediziner aus Florida vermuten einen Zusammenhang zwischen Arthritis und systemischer Entzündung bei älteren Männern und wollten darüber hinaus untersuchen, ob diese Assoziationen durch eine schlechtere Schlafgesundheit vermittelt werden.

Die Wissenschaftlerinnen und Wissenschaftler griffen auf Daten der MrOS-Studie (Osteoporotic Fractures in Men Study) zu. Es handelt sich um eine prospektive Studie mit knapp 6000 Männern, die zwischen 2000 und 2002 in 6 geografisch unterschiedlichen Regionen der USA, rekrutiert wurden. Dabei mussten die Männer 65 Jahre oder älter sein, selbstständig ohne Hilfen gehen können und durften keine beidseitigen Hüftgelenksprothesen haben.

Für die vorliegende Untersuchung wählte das Autorenteam eine Subgruppe aus, die zwischen 2003 und 2005 an einer ergänzenden Schlafstudie teilgenommen hatte. Für die Auswertung konstruierten die Forscher den zusammengesetzten Score einer mehrdimensionalen Schlafgesundheit aus wahrgenommener Schlafqualität, Schläf- 
rigkeit, Häufigkeit eines Nickerchens am Tag, Aufwachen nach Schlafbeginn und Schlafdauer. Diese Parameter wurden durch Eigenberichte der Patienten sowie durch Aktigrafie gemessen. Ebenso erstellten sie binäre Indikatoren einer erhöhten Inflammation anhand von C-reaktivem Protein (>3 mg/l) und Interleukin-6 (>1,08 pg/ml). Die Analysen erfolgten alterskontrolliert, anhand diagnostizierter Schlafstörungen, dem Body-Mass-Index, dem Raucherstatus sowie anhand des relevanten Medikamentengebrauchs und der Komorbiditäten.

In der ausgewählten Gruppe von 2562 Männern berichteten $24 \%$ über eine Arthrose und $8 \%$ über rheumatoide Arthritis. Von diesen RA-Patienten nahmen 0,7\% Antirheumatika ein. Die in der Stichprobe gefundenen Zahlen entsprachen der nationalen Prävalenzrate. Das mediane Alter der Kohorte lag bei $76 \pm 5,5$ Jahren.

Ältere Männer mit Arthrose (OA) hatten kein höheres Risiko für erhöhte CRP- oder IL-6-Spiegel, ganz im Gegensatz zu Männern mit rheumatoide Arthritis, bei denen eine höhere Wahrscheinlichkeit für erhöhte CRP- und IL-6-Spiegel festgestellt wurde.

Einen Zusammenhang zwischen OA und erhöhten CRP- oder IL-6-Spiegel auf Grund einer schlechteren Schlafgesundheit war indirekt nachweisbar. Denn OA war mit einer schlechteren Schlafgesundheit assoziiert, was weiterführend mit einer um $16 \%$ höheren Wahrscheinlichkeit eines erhöhten CRP $(p<0,001)$ und einer um $12 \%$ höheren Wahrscheinlichkeit eines erhöhten IL6 -Spiegels $(p<0,01)$ verbunden war. Diese Assoziation, von durch die Schlafgesundheit vermittelte erhöhte CRP- und IL-6-Spiegel, fand sich bei den Männern mit RA hingegen nicht.

\section{FAZIT}

Nach Meinung der Studienautorinnen und Autoren belegen die Ergebnisse, dass eine Förderung der Schlafgesundheit dazu beitragen kann, das Risiko einer systemischen Entzündung bei älteren Männern mit OA zu verringern. 